\title{
Face Recognition based on Weber Symmetrical Local Graph Structure
}

\author{
Jucheng Yang ${ }^{1}$, Lingchao Zhang ${ }^{1}$, Yuan Wang ${ }^{1}$, Tingting Zhao ${ }^{1 *}$, Wenhui Sun ${ }^{1}$ \\ and Dong Sun Park ${ }^{2}$ \\ ${ }^{1}$ College of Computer Science and Information Engineering Tianjin University of Science and Technology, \\ Tianjin, China. \\ [e-mail: tingting@tust.edu.cn] \\ ${ }^{2}$ Department of Electronic and Information Engineering, Chonbuk National University, \\ Jeonbuk, Republic of Korea. \\ [e-mail: dspark@jbnu.ac.kr] \\ * Corresponding author: Tingting Zhao
}

Received July 25, 2017; revised October 8, 2017; accepted November 29, 2017;

published April 30, 2018

\begin{abstract}
Weber Local Descriptor (WLD) is a stable and effective feature extraction algorithm, which is based on Weber's Law. It calculates the differential excitation information and direction information, and then integrates them to get the feature information of the image. However, WLD only considers the center pixel and its contrast with its surrounding pixels when calculating the differential excitation information. As a result, the illumination variation is relatively sensitive, and the selection of the neighbor area is rather small. This may make the whole information is divided into small pieces, thus, it is difficult to be recognized. In order to overcome this problem, this paper proposes Weber Symmetrical Local Graph Structure (WSLGS), which constructs the graph structure based on the $5 \times 5$ neighborhood. Then the information obtained is regarded as the differential excitation information. Finally, we demonstrate the effectiveness of our proposed method on the database of ORL, JAFFE and our own built database, high-definition infrared faces. The experimental results show that WSLGS provides higher recognition rate and shorter image processing time compared with traditional algorithms.
\end{abstract}

Keywords: Face Recognition, Weber Symmetric Local Graph Structure, Local Graph Structure, Weber Local Descriptor, Local Binary Pattern 


\section{Introduction}

A utomatic human identification is one of the interesting categories in biometric information which has attracted much attention recently [1]. Face recognition is one important way of human identification due to the way of non-contact acquisition and friendly interactive interface. Face recognition technology can be applied in a wide range of fields, such as identity authentication, access control and so on. The goal is to identify faces in a still image or video regardless of variations such as pose, illumination, occlusion and expression.

A complete face recognition system includes three modules, i.e. face detection, feature extraction and face matching [2]. Feature extraction is an important step. It can overcome the influence from different environments and extract the representative features which have a great impact on the recognition rates. Over the past few decades, many algorithms are proposed to extract the facial features.

In 1994, Local Binary Pattern was proposed [3], which describes the local texture information of the image and extracts features by comparing the center pixel value with its surrounding pixel value. Subsequently, many local algorithms have been proposed on the basis of LBP. The CS-LBP [4] operator proposed by Heikkilä et al. and the DLBP [5] operator proposed by Yang et al. have achieved good results. Weber Local Descriptor (WLD) is a stable and effective feature extraction algorithm [6], which was proposed by Chen Jie et al.. The operator calculates image differential excitation and direction information, and integrates them together. After that, Abusham [7] et al. employed the concept of graph structure for feature extraction and proposed the algorithm of Local Graph Structure (LGS), which has been demonstrated with good performance. In 2014, Mohd et al. [8] proposed a Symmetrical Local Graph Structure (SLGS) algorithm, which took balanced advantage of the pixels in the right side and left side of target pixel.

This paper proposes the algorithm of Weber Symmetrical Local Graph Structure (WSLGS), which is inspired by WLD, LGS and SLGS. The algorithm is based on the neighborhood of $5 \times 5$. It constructs symmetric graph structure in two diagonals directions based on the original LGS algorithm and SLGS algorithm, and see it as the differential excitation in the WSLGS algorithm. Then, the differential excitation information is integrated with the information of the WLD algorithm to get the features of the center pixel. In the implementation, we apply principle component analysis (PCA) [9] [10] to reduce the dimensionality of the feature matrix, where the principal component contribution rate is set to 0.95. Then, we employ Extreme Learning Machine (ELM) [11] [12] to classify the images. Through the experiments conducted on ORL, JAFFE and high-definition infrared face database which is built by ourselves, we conclude that the recognition rate of WSLGS algorithm and the time of image processing are better than the traditional algorithms.

The rest of this article is organized as follows. The second section reviews the algorithms of WLD and LGS; Section 3 gives the detailed information of our proposed algorithm, WSLGS; Section 4 shows the experimental results on ORL, JAFFE and high definition infrared face database; Finally, we conclude this manuscript in Section 5. 


\section{Related Work}

\subsection{Weber Local Descriptor}

The idea of WLD operator is inspired by Weber's law [13]. The content of Weber's law is that the difference threshold of sensation changes regularly with the change of the original stimulus. The regularity can be expressed as:

$$
\frac{\Delta I}{I}=k
$$

$I$ is the background intensity, $\Delta I$ is the perceptual increment threshold. And $k$ is a Weber fraction, which is set as a constant.

According to Weber's law, Chen Jie et al. introduced the differential stimulus and the direction of two stimulus in the description of image texture [6]. The differential excitation value is obtained by calculating the ratio of the center pixel value and the difference between center pixel and its surrounding pixels in the $3 \times 3$ neighborhood, which can be formulated as:

$$
W L D\left(x_{c}\right)=\arctan \left[\sum_{i=0}^{P-1}\left(\frac{x_{i}-x_{c}}{x_{c}}\right)\right]
$$

where $P$ is the size of neighborhood area, which is $9 . x_{c}$ represents the central pixel and $x_{i}$ represents the surrounding pixels.

The direction is the gradients of the image in the direction of horizontal and vertical, which is denoted as:

$$
W L D\left(x_{c}\right)=\arctan \left[\alpha \sum_{i=0}^{P-1}\left(\frac{x_{i}-x_{c}}{x_{c}+\lambda}\right)\right]
$$

where $\alpha$ can be used to magnify or shrink the difference between neighbors. $\lambda$ is assigned as a small constant value to avoid the situation of zero denominator.

In 2013, Li et al. [14] used the WLD operator for face recognition and achieved good results.

\subsection{Local Graph Structure}

The Local Graph Structure (LGS) operator considers the structure of graph, which constructs the graph within the neighborhood of $4 \times 3$, and compares the values with its adjacent pixels. The comparison results are noted by the binary value. Finally, convert it to the format of decimal number.

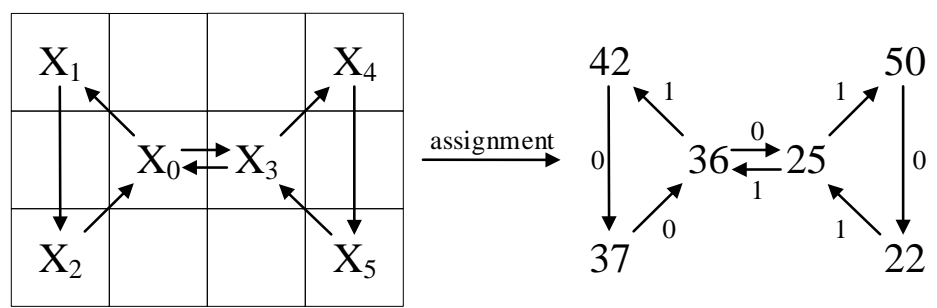

Fig. 1. Template of LGS operator

An example of LGS operator is shown in Fig. 1, where $X_{0}$ as the target pixel. By comparing the value of the pixel with its adjacent pixel along the direction of arrows, the binary value of $\mathrm{X}_{0}$ is 10001011 . As a result, the decimal vale of the target pixel is obtained as 139 . 
In 2012, Abusham et al. [8] used the LGS operator for face recognition and achieved good results.

\section{Proposed Method}

\subsection{WSLGS}

Although the success of the WLD and LGS algorithm in image feature extraction have been demonstrated, there are still many problems. The differential excitation in the algorithm of WLD only considers the contrast information between the center pixel and the surrounding pixels, which is not enough to represent the complete information of the image. On the other hand, the LGS algorithm transforms the characteristic values of the central pixels from binary to decimal format in the process of calculation, which leads to the loss of information. In order to solve these problems, we propose Weber Symmetrical Local Graph Structure algorithm, which combines these two algorithms. More specifically, WSLGS takes the $5 \times 5$ neighborhood, constructs the graph structure on two diagonal directions of the center pixel, and calculates the sum of the difference of adjacent pixels in a certain order. Finally, the new differential excitation information is integrated with the original direction information in the WLD algorithm to represent the characteristic values of the center pixel.

The algorithm, Weber Symmetrical Local Graph Structure, is shown in the following formulas and graphs:

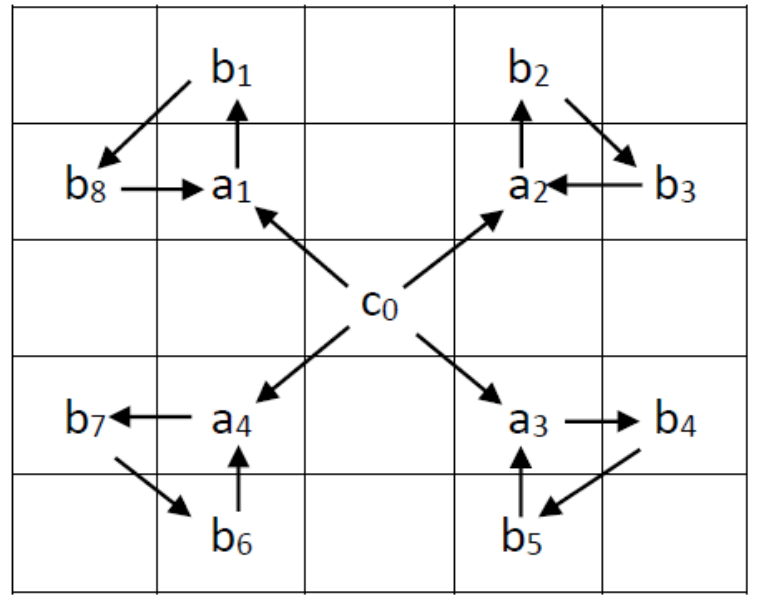

Fig. 2. WSLGS algorithm

$$
\begin{gathered}
\text { WSLGS }=\arctan \left(\alpha \frac{x_{g}}{x_{m}+\lambda}\right) \\
x_{1}=\left|c_{0}-a_{1}\right|+\left|a_{1}-b_{1}\right|+\left|b_{1}-b_{8}\right|+\left|b_{8}-a_{1}\right| \\
x_{2}=\left|c_{0}-a_{3}\right|+\left|a_{3}-b_{4}\right|+\left|b_{4}-b_{5}\right|+\left|b_{5}-a_{3}\right| \\
x_{3}=\left|c_{0}-a_{2}\right|+\left|a_{2}-b_{2}\right|+\left|b_{2}-b_{3}\right|+\left|b_{3}-a_{2}\right| \\
x_{4}=\left|c_{0}-a_{4}\right|+\left|a_{4}-b_{7}\right|+\left|b_{7}-b_{6}\right|+\left|b_{6}-a_{4}\right|
\end{gathered}
$$




$$
\begin{gathered}
x_{g}=x_{1}+x_{2}+x_{3}+x_{4} \\
x_{m}=\frac{1}{13}\left(c_{0}+\sum_{i=1}^{4} a_{i}+\sum_{j=1}^{8} b_{j}\right)
\end{gathered}
$$

where $x_{1}, x_{2}, x_{3}$ and $x_{4}$ are the sum of the differences between adjacent pixels in the northwest, southeast, northeast and southwest of the center pixel respectively. $x_{g}$ is the sum of the four directions, which is the sum of the differences between adjacent pixels in the graph constructed in two diagonal directions in the neighborhood, $x_{m}$ is the average value of the pixels involved in the graph structure. According to the empirical values, $\alpha$ and $\lambda$ were 3 and $1 \times 10^{-7}$, respectively [15].

Compared with LGS algorithm, the WSLGS algorithm makes up the information loss problem caused by the conversion between binary and decimal. Moreover, the constructed graph structure has a larger coverage and contains more accurate feature information compared to the SLGS. In addition, the final characteristic values include two kinds of information by applying of Weber's law, which are the differential excitation and the direction. Thus, the WSLGS algorithm is more robust and makes significant improvement in terms of recognition rate.

\subsection{Dimensionality Reduction}

After the feature extraction, the size of the feature matrix could be very large. In order to avoid curve of dimensionality, we use the method of principle component analysis (PCA) [9] [10] to implement the dimensionality reduction. The principal component contribution rate is set to 0.95 .

\subsection{Training and Classification}

After reducing the dimensionality of the feature data, we employ the Extreme Learning Machine [11] to classify. ELM is a kind of feedforward neural network algorithm proposed by Huang et al. [12]. Compared with the traditional classifier, it is not necessary to set the weight and bias of neuron nodes in each hidden layer, which has the advantages of simple calculation and fast learning speed. In our experiments, the activation function uses the sig function, and the number of hidden layer nodes is set to 2000 .

\section{Experiments and analysis}

\subsection{Experimental database}

Our experiments are performed on three databases, i.e., the ORL face database [16], our lab's high definition infrared face database and JAFFE face expression database [17].

ORL face database was created by the AT\&T Laboratory of University of Cambridge. It contains 40 people, each person is with 10 images, there are 400 face images in total. These images include changes in posture and expression. Specific examples are shown in Fig. 3. Our own built HD infrared face database contains 80 volunteers with different ages and gender totally. Each person has 9 photos, a total of 720 images. These images are collected from different angles. Specific examples are shown in Fig. 4. JAFFE database is an expression database created by Japan's ATR (Advanced Telecommunication Research Institute International). It is devoted to the study of facial expression recognition, including 10 individuals, each with 7 kinds of expressions. In the original database, the number of images 
per person is different, a total of 216 images. For the convenience of testing, we selected 20 images per person, a total of 200 images for the experiment. Specific examples are shown in Fig. 5.

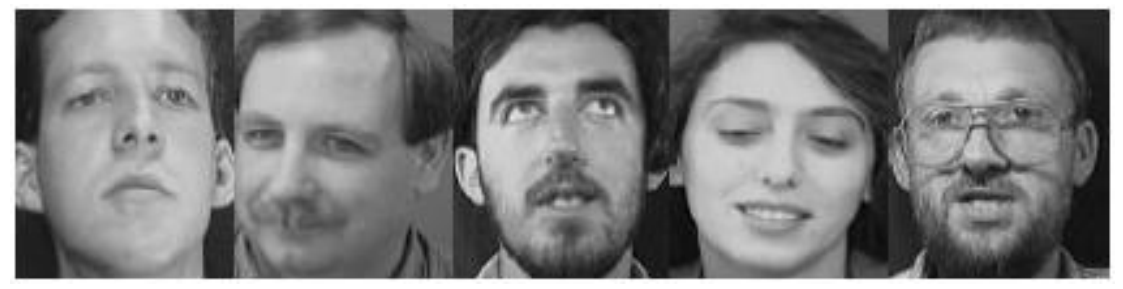

Fig. 3. Examples of ORL face database

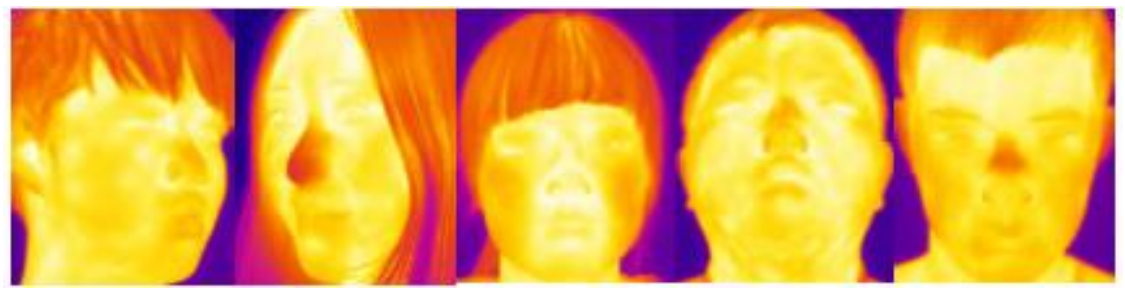

Fig. 4. Examples of HD infrared face database

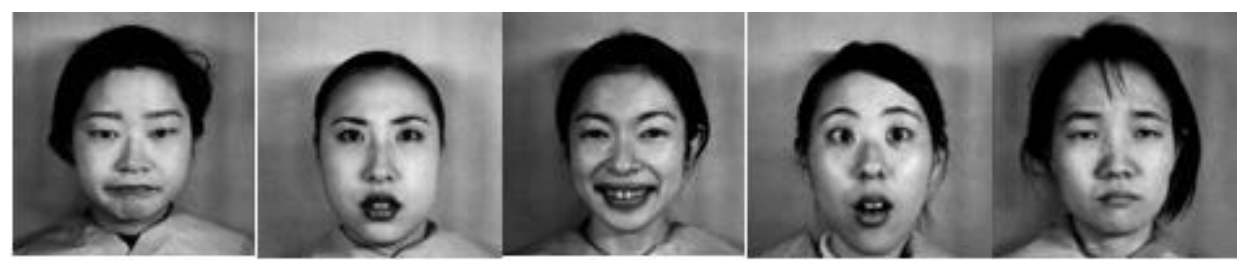

Fig. 5. Examples of JAFFE face database

\subsection{Experimental results on ORL face database}

We conducted experiments on the ORL face database firstly, and compare our proposed method WSLGS with LBP, LGS, WLD and SLGS. The number of training samples is 3,4,5,6, and the results are shown in Table.1. As can be seen from the table, the recognition rate of our proposed algorithm is higher than other algorithms under the same conditions. When the number of training samples is 6, the recognition rate using WSLGS algorithm can reach $100 \%$.

Table 1. Recognition rates of different algorithms on the ORL database

\begin{tabular}{|c|c|c|c|c|c|}
\hline $\begin{array}{c}\text { Number of training } \\
\text { samples(N) }\end{array}$ & LBP & LGS & WLD & SLGS & WSLGS \\
\hline 3 & 0.5911 & 0.5496 & 0.9382 & 0.5639 & 0.9518 \\
\hline 4 & 0.6687 & 0.6429 & 0.9600 & 0.6233 & 0.9854 \\
\hline 5 & 0.6665 & 0.6175 & 0.9670 & 0.6475 & 0.9890 \\
\hline 6 & 0.7150 & 0.6663 & 0.9919 & 0.6881 & 1.0000 \\
\hline
\end{tabular}

Fig. 6 shows the recognition rates of LBP, LGS, WLD, SLGS and WSLGS intuitively. As can be seen from it, the curve of WSLGS is above others, which means the WSLGS has the best recognition performance. 


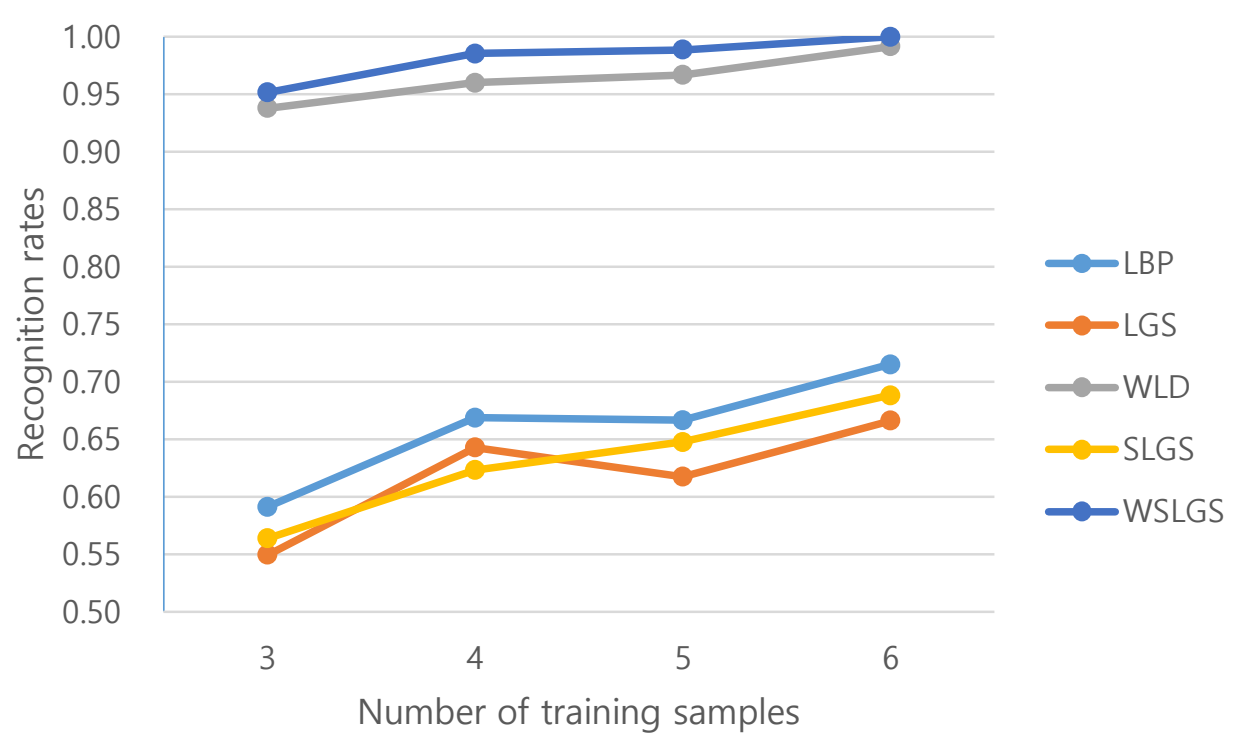

Fig. 6. Recognition rates of different algorithms on the ORL database

\subsection{Experimental results on HD infrared face database}

We also did experiments on the HD infrared face database which was built by ourselves. We compared WSLGS algorithm with LBP, LGS, WLD and SLGS. The number of training samples is 5,6,7,8, and the experimental results are shown in Table. 2. As can be seen from the table, the recognition rate of WSLGS algorithm is higher than the traditional algorithm under the same conditions. When the number of training samples is 8 , the recognition rate is up to $100 \%$. At the same time, the recognition rates of the other three algorithms are 54.37\%, $57.50 \%, 98.63 \%$ and $59.13 \%$.

Table 2. Recognition rates of different algorithms on the HD infrared face database

\begin{tabular}{|c|c|c|c|c|c|}
\hline $\begin{array}{c}\text { Number of training } \\
\text { samples(N) }\end{array}$ & LBP & LGS & WLD & SLGS & WSLGS \\
\hline 5 & 0.5516 & 0.4562 & 0.9200 & 0.3556 & 0.9350 \\
\hline 6 & 0.5375 & 0.5104 & 0.9575 & 0.4233 & 0.9771 \\
\hline 7 & 0.5250 & 0.5344 & 0.9769 & 0.4975 & 0.9881 \\
\hline 8 & 0.5437 & 0.5750 & 0.9863 & 0.5913 & 1.0000 \\
\hline
\end{tabular}

Fig. 7 is a line chart displaying the recognition rates of LBP, LGS, WLD, SLGS and WSLGS on the HD infrared face database. As we can see from it, WSLGS has the best performance because it has the best recognition rates no matter what the number of training samples used. 


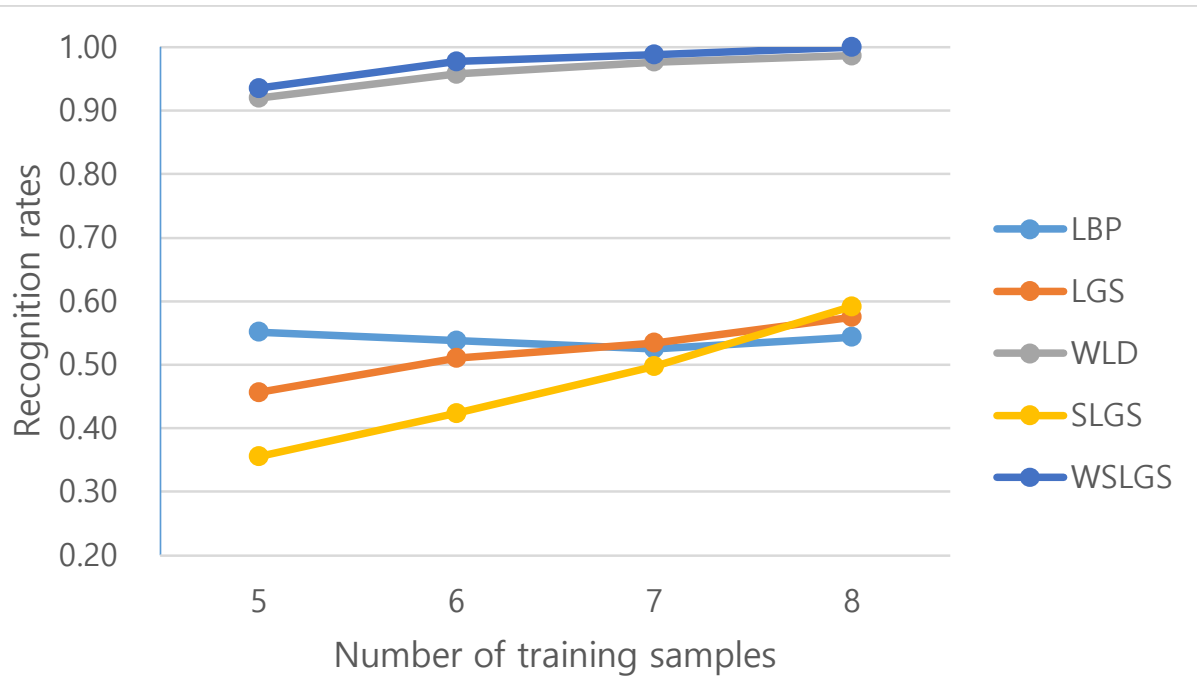

Fig.7. Recognition rates of different algorithms on the HD infrared face database

\subsection{Experimental results on JAFFE face database}

In order to verify the robustness of our proposed algorithm, we also completed the experiment on JAFFE, which was devoted to facial research. The training samples for the LBP, LGS, WLD, SLGS and WSLGS algorithms are selected as 3, 4, 5, 6, 7, 8, and the experimental results are shown in Table 3. It is clear from the table that the recognition rate of WSLGS algorithm is higher than the traditional algorithms. When the number of training samples is 8 , the recognition rate of LBP, LGS, WLD and SLGS is $92.08 \%, 84.25 \%, 91.75 \%$ and $88.67 \%$ respectively. And the recognition rate of our proposed method can reach $99.92 \%$.

Table 3. Recognition rates of different algorithms on the JAFFE face database

\begin{tabular}{|c|c|c|c|c|c|}
\hline $\begin{array}{c}\text { Number of training } \\
\text { samples(N) }\end{array}$ & LBP & LGS & WLD & SLGS & WSLGS \\
\hline 3 & 0.7947 & 0.6212 & 0.8471 & 0.7706 & 0.9000 \\
\hline 4 & 0.8563 & 0.7344 & 0.8913 & 0.8531 & 0.8988 \\
\hline 5 & 0.8620 & 0.7467 & 0.9120 & 0.8473 & 0.8967 \\
\hline 6 & 0.8600 & 0.7371 & 0.9221 & 0.8414 & 0.9357 \\
\hline 7 & 0.9031 & 0.8154 & 0.9192 & 0.8792 & 0.9415 \\
\hline 8 & 0.9208 & 0.8425 & 0.9175 & 0.8867 & 0.9992 \\
\hline
\end{tabular}

Fig. 8 shows the recognition rate of each algorithm using a line chart. We can clearly see that the WSLGS algorithm is located at the top of the line chart, which means that compared with the traditional algorithm, WSLGS algorithm has the best performance. 


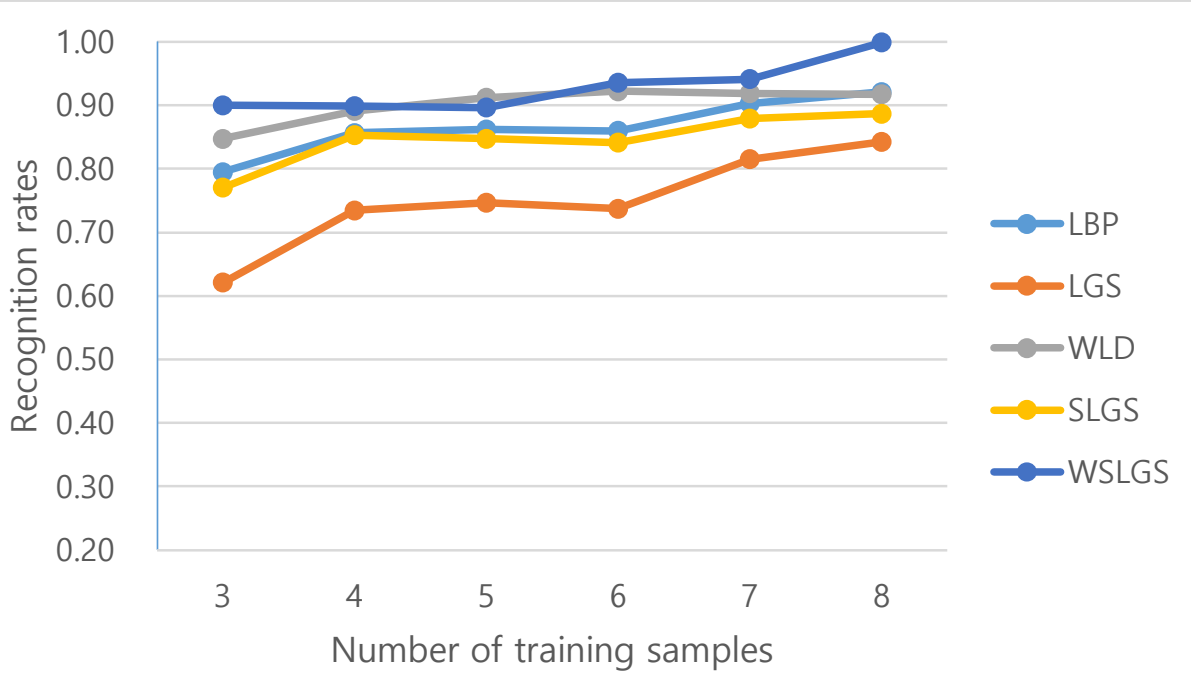

Fig. 8. Recognition rates of different algorithms on the JAFFE face database

\subsection{ROC curves}

In order to further investigate the superiority of our proposed method, we carry out experiments on the same database and draw the ROC curves as shown in Fig. 9.

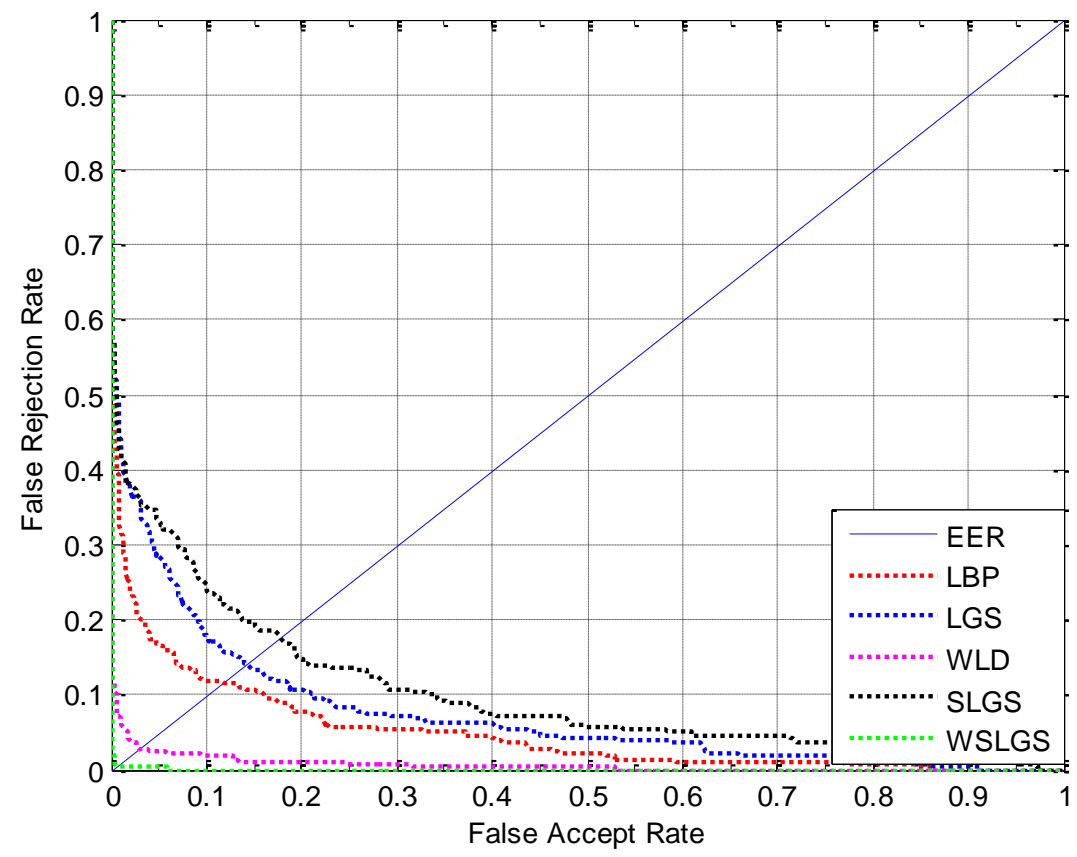

Fig. 9. ROC curves of different algorithms

The smaller EER, the higher overall performance of algorithms. The above diagram represents the ROC curve of different algorithms. We can realize from it that the EER of the LBP is approximately 0.12 , the LGS is approximately 0.15 , the SLGS is approximately 0.18 , the WLD is approximately 0.04 , but our WSLGS is less than 0.01 . It's clearly seen that our 
algorithm's curve is located below the traditional algorithms which means the identification error of WSLGS is the smallest and the recognition rate is the best.

\subsection{Comparison of the processing time}

In addition to the comparison of the recognition rate, we also test the processing time of the same image with the methods of LBP, LGS, WLD, SLGS and proposed method WSLGS. The experimental results are shown in Table 4. As can be seen from the table, the WSLGS algorithm requires the shortest time to process an image.

Table 4. The Processing time of different algorithms

\begin{tabular}{|c|c|c|c|c|c|}
\hline Algorithms & LBP & LGS & WLD & SLGS & WSLGS \\
\hline $\begin{array}{c}\text { Processing } \\
\text { time(second) }\end{array}$ & 0.2839 & 0.2917 & 0.0640 & 0.2948 & 0.0530 \\
\hline
\end{tabular}

\section{Conclusion}

In this paper, we proposed Weber Symmetrical Local Graph Structure, which is used to extract the features of face images. This algorithm constructs the graph structure from the diagonal direction in the neighborhood of $5 \times 5$, and then combines it with the direction with the differential excitation information according to Weber's law. Finally, the eigenvalues of the image are obtained. The overall structure used in the calculation of eigenvalues is more suitable for human faces, and the $5 \times 5$ neighborhoods can also ensure that some of the more obvious overall features of the face are not too small to be undetectable. So WSLGS algorithm is more suitable for face recognition than traditional algorithms. We implemented the algorithm in the ORL database, our own built high definition infrared face database and JAFFE database. The experimental results demonstrated that the recognition rate of WSLGS algorithm is better than the traditional algorithms, and the processing time is lower than the traditional algorithms. In the future, we will try to further improve the WSLGS algorithm, hoping to be applied to other areas of biometrics, such as finger vein recognition.

\section{References}

[1] W. Zhao, R. Chellappa, P. J. Phillips, and A. Rosenfeld, "Face recognition: A literature survey," ACM computing surveys (CSUR), vol. 35, no. 4, pp. 399-458, 2003. Article (CrossRef Link)

[2] H. Yu and J. Yang, "A direct LDA algorithm for high-dimensional data - with application to face recognition," Pattern recognition, vol. 34, no. 10, pp. 2067-2070, 2001. Article (CrossRef Link)

[3] Ojala, Timo, Matti Pietikainen, and David Harwood. "Performance evaluation of texture measures with classification based on Kullback discrimination of distributions," in Proc. of Pattern Recognition, 1994. Vol. 1-Conference A: Computer Vision \& Image Processing., Proceedings of the 12th IAPR International Conference on. Vol. 1. IEEE, vol. 1, pp. 582-585, 1994.

Article (CrossRef Link)

[4] M. Heikkilä, M. Pietikäinen, and C. Schmid, "Description of interest regions with local binary patterns," Pattern recognition, vol. 42, no. 3, pp. 425-436, 2009. Article (CrossRef Link)

[5] X. Yang, X. Gao, and Q. Tian, "Polar embedding for aurora image retrieval," IEEE Transactions on Image Processing, vol. 24, no. 11, pp. 3332-3344, 2015.

Article (CrossRef Link) 
[6] J. Chen et al., "WLD: A robust local image descriptor," IEEE transactions on pattern analysis and machine intelligence, vol. 32, no. 9, pp. 1705-1720, 2010. Article (CrossRef Link)

[7] Abusham, Eimad EA, and Housam K. Bashir. "Face recognition using local graph structure (LGS)," in Proc. of International Conference on Human-Computer Interaction. Springer, Berlin, Heidelberg, pp. 169-175, 2011. Article (CrossRef Link)

[8] M. F. A. Abdullah, M. S. Sayeed, K. S. Muthu, H. K. Bashier, A. Azman, and S. Z. Ibrahim, "Face recognition with symmetric local graph structure (slgs)," Expert Systems with Applications, vol. 41, no. 14, pp. 6131-6137, 2014. Article (CrossRef Link)

[9] Y. Luo, T. Zhang, and Y. Zhang, "A novel fusion method of PCA and LDP for facial expression feature extraction," Optik-International Journal for Light and Electron Optics, vol. 127, no. 2, pp. 718-721, 2016. Article (CrossRef Link)

[10] M. Turk and A. Pentland, "Eigenfaces for recognition," Journal of cognitive neuroscience, vol. 3, no. 1, pp. 71-86, 1991. Article (CrossRef Link)

[11] G.-B. Huang, Q.-Y. Zhu, and C.-K. Siew, "Extreme learning machine: theory and applications," Neurocomputing, vol. 70, no. 1, pp. 489-501, 2006. Article (CrossRef Link)

[12] W. Zong and G.-B. Huang, "Face recognition based on extreme learning machine," Neurocomputing, vol. 74, no. 16, pp. 2541-2551, 2011. Article (CrossRef Link)

[13] Chen, Jie, et al. "A robust descriptor based on weber's law," in Proc. of Computer Vision and Pattern Recognition, 2008. CVPR 2008. IEEE Conference on. IEEE, pp. 1-7, 2008. Article (CrossRef Link)

[14] S. Li, D. Gong, and Y. Yuan, "Face recognition using Weber local descriptors," Neurocomputing, vol. 122, pp. 272-283, 2013. Article (CrossRef Link)

[15] B. Wang, W. Li, W. Yang, and Q. Liao, "Illumination normalization based on weber's law with application to face recognition," IEEE Signal Processing Letters, vol. 18, no. 8, pp. 462-465, 2011. Article (CrossRef Link)

[16] F. S. Samaria and A. C. Harter, "Parameterisation of a stochastic model for human face identification," in Proc. of Proceedings of the Second IEEE Workshop on, presented at the Applications of Computer Vision, 1994., pp. 138-142, 1994. Article (CrossRef Link)

[17] M. J. Lyons, J. Budynek, and S. Akamatsu, "Automatic classification of single facial images," IEEE transactions on pattern analysis and machine intelligence, vol. 21, no. 12, pp. 1357-1362, 1999. Article (CrossRef Link)

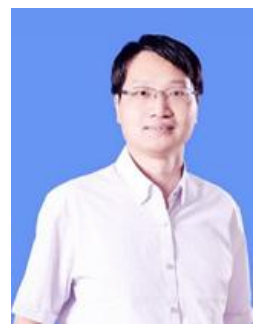

Jucheng Yang is a professor with the College of Computer Science and Information Engineering, Tianjin University of Science and Technology, Tianjin, P.R. China. He received his B.S. degree from South-Central University for Nationalities, China, M.S. and Ph.D. degrees from Chonbuk National University, Republic of Korea. He did his postdoctoral work at the Advanced Graduate Education Center of Jeonbuk for Electronics and Information Technology-BK21 (AGECJEIT-BK21) in Chonbuk National University. He has published over 80 papers in related international journals and conferences, such as IEEE Trans. on HMS, Expert Systems with Applications, IEEE Systems Journal and so on. He has served as editor of five books in biometrics (Intech publisher). He owns seven patents in biometrics. His research interests include image processing, biometrics, pattern recognition, and neural networks.

E-mail: jcyang@tust.edu.cn. 

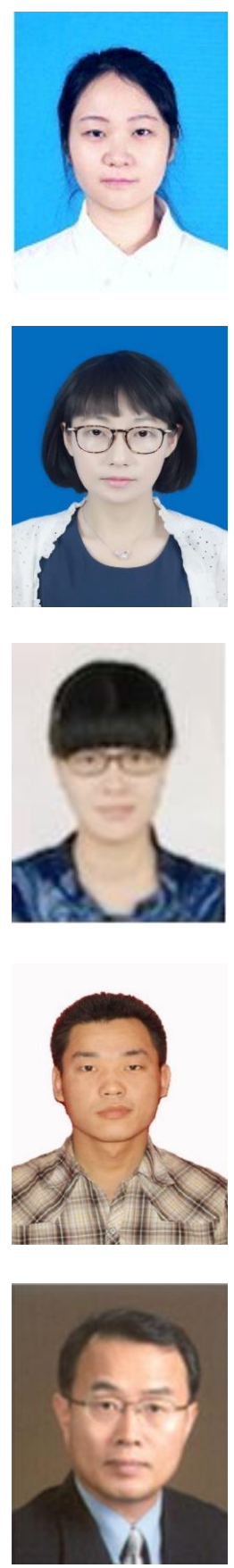

Lingchao Zhang is a master student in the College of Computer Science and Information Engineering, Tianjin University of Science and Technology, Tianjin, P.R. China. She received her B.S. degree from Tianjin University of Science and Technology, China, 2016. Her research interests include image processing, biometrics, pattern recognition, and neural networks.

E-mail:zhanglc@mail.tust.edu.cn.

Yuan Wang is an assistant professor in the College of Computer Science and Information Engineering, Tianjin University of Science and Technology, Tianjin P.R.China. She has received her Ph.D. degree in computer science at Nankai University, Tianjin China, in 2016. She has published papers in top conferences and journals, inlcuding AAAI, ICDE, WWW and TKDE. Her current research interests include machine learning, web search, social media and natural language understanding.

E-mail: wangyuan23@tust.edu.cn.

Tingting Zhao, Ph.D., associate professor in College of Computer Science and Information Engineering, Tianjin University of Science and Technology, Tianjin, P.R. China.. She published more than 20 papers about machine learning algorithms in top journals and conferences in the field of machine learning. Her main research interests include artificial intelligence and machine learning.

E-mail: tingting@tust.edu.cn.

Wenhui Sun is a master student in the College of Computer Science and Information Engineering, Tianjin University of Science and Technology, Tianjin, P.R. China. He received his B.S. degree from Taiyuan University of Science and Technology, China, 2016. His research interests include image processing, biometrics, pattern recognition, and neural networks.

E-mail:122992564@qq.com.

Dong Sun Park is a Professor with the Department of Electronics Engineering at Chonbuk National University in South Korea. He received his B.S. degree from the Department of Electronic Engineering of Korea University, South Korea in 1979, and his M.S. and Ph.D. degrees from University of Missouri, USA, in 1984 and 1991 respectively. His research interests include deep neural networks, pattern recognition, image processing, digital systems design.

E-mail: dspark@jbnu.ac.kr. 